\title{
Zygomatic Implant-Supported Prosthesis: When the Prosthetic Rehabilitation Affects the Function: A Case Report
}

\author{
Wilson Matsumoto ${ }^{{ }^{*}}$, Rossana Pereira De Almeida ${ }^{1}$, Alexandre Elias Trivellato ${ }^{2}$, \\ Cassio Edvard Sverzut ${ }^{2}$, Takami Hirono Hotta ${ }^{1}$
}

${ }^{1}$ Dental Materials and Prosthodontic Department, Dental School of Ribeirão Preto, University of São Paulo, São Paulo, Brazil ${ }^{2}$ Oral and Maxillofacial Surgery Traumatology and Periodontology Department, Dental School of Ribeirão Preto, University of São Paulo, São Paulo, Brazil

Email: *wmatsumoto@forp.usp.br, rpaa@forp.usp.br, eliastrivellato@forp.usp.br, cesve@forp.usp.br, takamihotta@forp.usp.br

How to cite this paper: Matsumoto, W., De Almeida, R.P., Trivellato, A.E., Sverzut, C.E. and Hotta, T.H. (2017) Zygomatic Implant-Supported Prosthesis: When the Prosthetic Rehabilitation Affects the Function: A Case Report. Open Journal of Stomatology, 7, 448-454.

https://doi.org/10.4236/ojst.2017.710039

Received: August 30, 2017

Accepted: October 9, 2017

Published: October 12, 2017

Copyright (c) 2017 by authors and Scientific Research Publishing Inc. This work is licensed under the Creative Commons Attribution International License (CC BY 4.0).

http://creativecommons.org/licenses/by/4.0/

Open Access

\begin{abstract}
The oral rehabilitation of edentulous patients can be done in different ways depending on the alveolar ridge morphology and patient expectations. The objective of this clinical case was to report prosthetic and functional adaptation difficulties when conventional complete denture was changed to a zygomatic implant-supported prosthesis. We report a 52-year-old male singer with an atrophic maxilla who was rehabilitated with zygomatic implant-supported prosthesis using 4 implants. However, the thickness of the prosthesis in the palatal region and the space between the prosthesis and soft tissue caused difficulty in speaking and singing. The palatal region of the prosthesis was trimmed and the anterior region of the prosthesis was relined. These procedures were performed to make room for the tongue and minimize the passage of air during speech. Adaptations in the shape of the prosthesis must be made to enable its use, without compromising its strength.
\end{abstract}

\section{Keywords}

Zygomatic Implant, Branemark Protocol, Implant Prosthesis

\section{Introduction}

Tooth loss causes physical, emotional [1] and behavioral disorders [2]. It also affects the functional aspects related to the pattern and efficiency of mastication and phonetics.

Oral rehabilitation of edentulous patients requires careful considerations of 
their age, esthetics, ease of cleaning, psychological status, degree of bone resorption, anatomic limitations, interocclusal space and cost-effectiveness [2]. The oral rehabilitation should meet all or the vast majority of patient expectations, including esthetic. However, physical and mechanical requirements must not be compromised to avoid change in the resistance, retention, and function of the prosthesis.

Zygomatic implants have been indicated for severely atrophic maxilla and have shown promising outcomes [1] [3] [4] [5] allowing anchorage and stability in the zygomatic bone, that is characterized by a cortical bone with a dense trabecular structure [6]. Zygomatic implant placement is a less invasive technique when compared to bone grafting. The grafting procedures increase the number of surgical procedures, overall cost, and treatment duration, causing severe discomfort for the patients [4] [7] [8].

The original placement technique recommends that the zygomatic implants should be placed through the maxillary sinus and anchored in the zygomatic bone [9], however, different fixation techniques of the implants were developed to improve the positioning with respect to the alveolar ridge [1] [7] [10] favoring the prosthetic rehabilitation.

The surgical and prosthetic resolution should allow patients similar biomechanical conditions to their natural teeth [10]. This resolution is not possible every time because there may be limitations imposed by the characteristics presented by patients.

Therefore, we present a report of a zygomatic implant-supported prosthesis, in a complete denture patient who had difficulties in singing and talking.

\section{Case Report}

A 52-year-old male, singer, presented for treatment at the clinic of Dental School of Ribeirao Preto with complaints about zygomatic implants rehabilitation done 8 months before. Clinical examination revealed a completely edentulous patient who had severe atrophy of the maxilla with extensive resorption of the alveolar bone, loss of lip support, and four zygomatic implants that were placed in the maxillary cuspid and premolar regions with healing abutments positioned (Figure 1). The original Branemark et al. (2004) surgical technique was used to place the zygomatic implants [9]. Figure 2 shows the panoramic radiography after the zygomatic implant placements.

The conventional total denture needed to be adjusted over the healing abutments. Small portions of zinc-enolic paste catalyst were put over the healing abutments and the denture was inserted in the oral cavity in order to mark position of the healing abutments on the prosthesis. In these points were made perforations with a tungsten carbide bur to allow adaptation of the denture over the healing abutments while maintaining the correct seating on the support areas. Perforations were sealed with autopolymerizing acrylic resin conforming to the contour of the healing abutments that allowed the adaptation and use of the denture (Figure 3). 


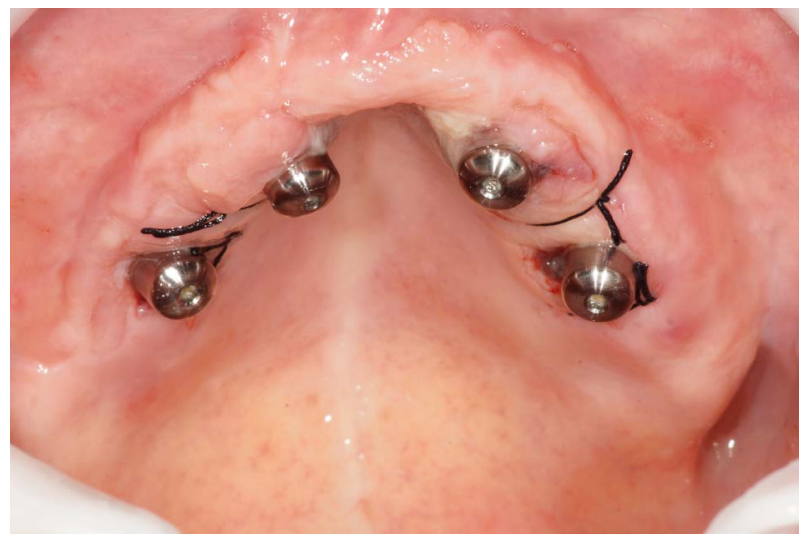

Figure 1. Complete edentulism with severe atrophy of maxilla and extensive resorption into the basal bone, high arched palate, and 04 zygomatic implants.

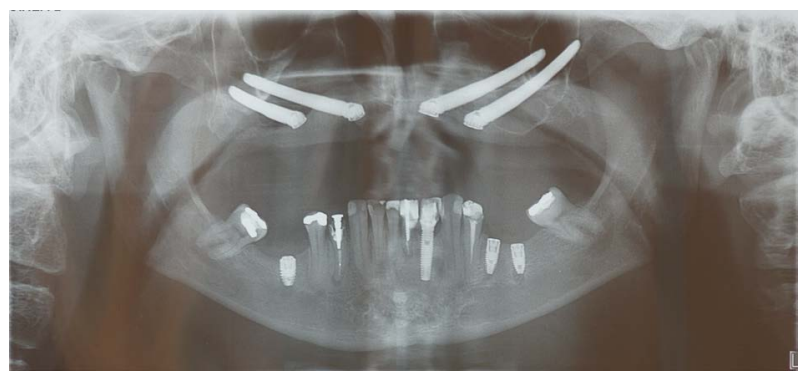

Figure 2. Panoramic radiography after the zygomatic implant placement.

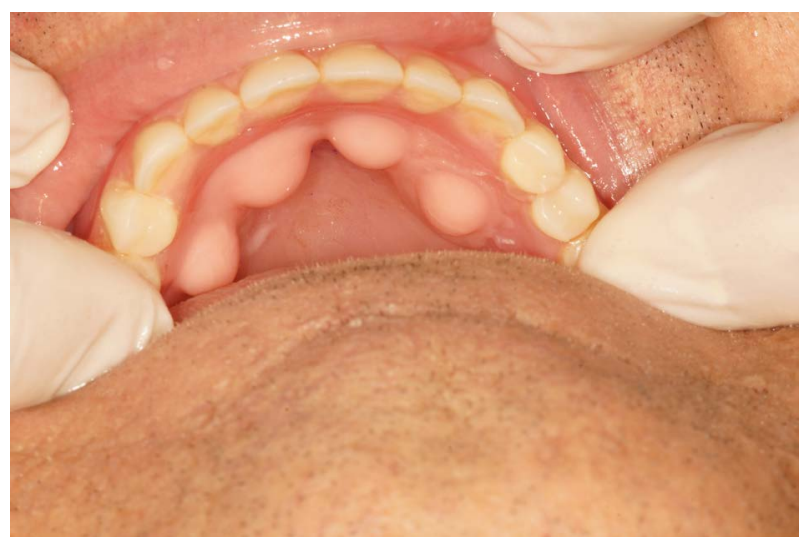

Figure 3. Relining of prosthesis to allow adaptation and use over the healing abutments.

Impressions of the implants and the residual ridge were made for the abutment selection. After installing the abutments, open-tray transfers were adapted and splinted with acrylic-resin, and an impression was made with a plastic tray using silicone impression material. Working cast was obtained and a multi-functional guide was fabricated using these models. The maxillomandibular relation was recorded using this multi-functional guide. The framework was fabricated using nickel-chromium alloy. Over this framework, artificial teeth were 
mounted and after esthetic and functional try-ins the prosthesis was finalized.

After a two weeks period of use of the prosthesis, the patient reported difficulty in speaking in addition to air leakage and change in the voice's tone. Then, the relining of the prosthesis was made to seal the space between it and the soft tissue.

The prosthesis was removed and cleaned, and a polyether adhesive layer was applied and an impression using polyether impression material was taken (Figure 4). The working cast obtained (Figure 5) was sent to a laboratory to fill-up the space using thermo-polymerizing acrylic resin. Figure 6 shows the prosthesis after the anterior space was filled-up with acrylic resin.

\section{Discussion}

Zygomatic implants have been indicated for severely atrophic maxilla and have shown promising outcomes [1] [3] [4] [5]. However, depending on the surgical technique, the implants can be positioned palatally, making the prosthetic rehabilitation difficult.

The use of the temporary complete denture is important for the patient that was treated with zygomatic implants because this maintains acceptable esthetics, masticatory and speech function during the healing process, and also explores the occlusal and esthetic position of teeth [3]. However, this temporary prosthesis presented different form from the definitive prosthesis due to the presence of the buccal flange that prevented the air leakage. In the present case report, after a period of two weeks with the interim complete prosthesis, the patient reported increased chewing ability but due to increased thickness in palatal region he reported difficulty speaking and singing.

A disadvantage of the technique utilized for the placement of zygomatic implants is the palatal implant emergence, which causes a reduction of the distance between the implants [1], reduced space for the tongue and the difficulties presented by the patient probably occurred due to necessity for the tongue's adaptation to such modifications.

When the Branemark protocol prosthesis was fitted and, at first, the patient's expectations for esthetic, adaptation, stability, and retention were successfull.

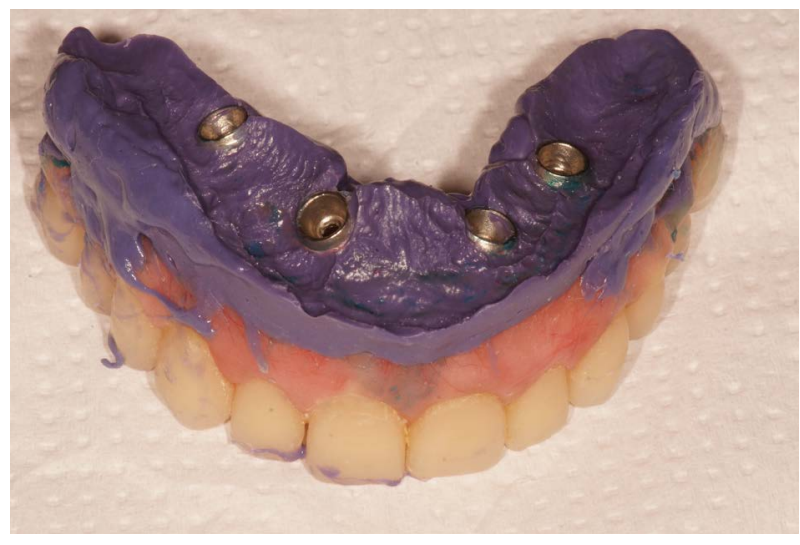

Figure 4. Impression using polyether material. 


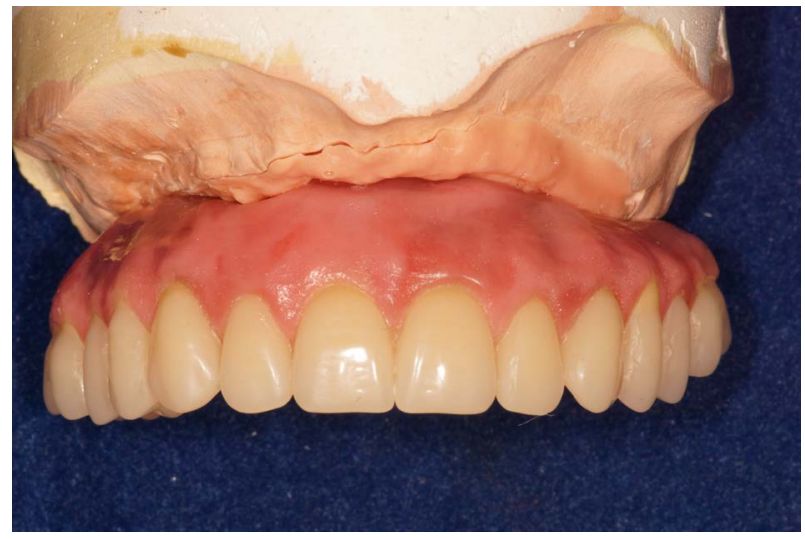

Figure 5. Working cast and relining of the prosthesis.

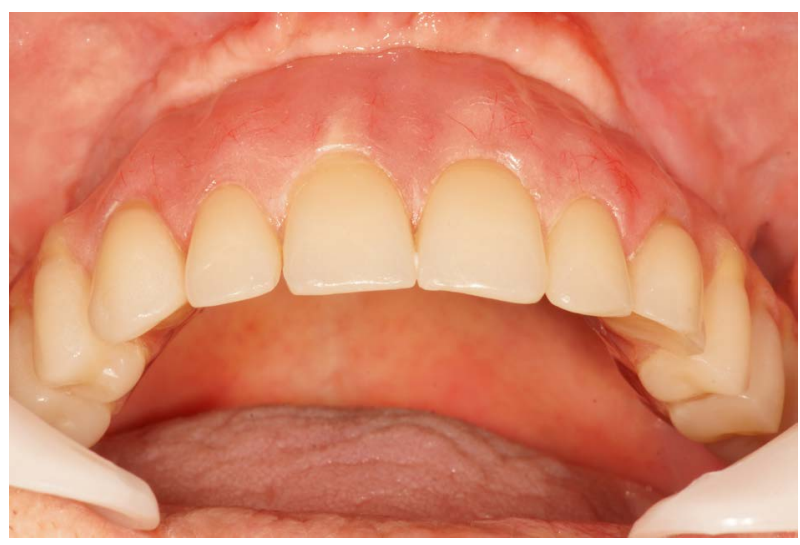

Figure 6. Prosthesis after the completion of the anterior space with acrylic resin.

But during follow-up, the patient continued to report difficulty in speaking and singing and also complaining of air leakage and loss of tone in his voice.

When the prosthesis is fitted, teeth position, palatal contours, occlusal vertical dimension and flange thickness can affect or interfere with speech articulation and intelligibility [2]. In this specific case of the implant-supported screw-retained prosthesis unlike the conventional complete denture intimate contact of the flange extension with the labial sulcus is not present therefore air may escape through the space between the prosthesis and gingiva, changing the speech of the individual as in the case of the patient who uses his voice professionally and this represents a significant disorder for him. However, this space is necessary and essential to the cleansing of the area and for the maintenance of healthy tissues.

During treatment planning every advantage and disadvantage must be explained to the patient so that the patient is aware of and understands the limitations of each treatment, especially if some expectation is not met. In this case, the patient's voice was the work tool and the palatal conformation, severely resorbed maxilla and zygomatic implant position contributed to potentiate the problems with speech. 
The resorption pattern of the maxilla and the selected surgical technique required the placement of the zygomatic implant in the palatal region of the resorbed posterior maxilla. In some cases, this prominence can disturb the patient's tongue but this discomfort should be transient and tolerable [11]. However, some researchers believe that the palatal zygomatic implant position causes neither discomfort nor problems in speech [9]. Although oriented on the possibility of problems in the voice, neither the professional nor the patient had experienced the extension of the problems in an individual who the voice is used as work tool. In this case it was a problem for the patient because he needs to use his voice in a more precise and refined way.

Because of this difficulty many visits for prosthetic adjustments were made, in order to reduce the thickness of the prosthesis in the palatal region but without compromising its resistance. However, air escape made it impossible for the patient to sing perfectly. The decision was then made fill-up the anterior space using acrylic resin even though the access at mucosal interface to fulfill hygienic demands had become more difficult. As cleaning is more difficult on the palatal side requiring reinforcement and modification of plaque control and home care maintenance [4]. Therefore, it was explained to the patient that this modification would require more effort in hygiene maintenance and more frequently periodic visits would be required

The functional result of this added resin was satisfactory to the patient. Even though the initial anterior pattern was not achieved he was able to return to his normal vocal activities.

\section{Conclusion}

Therefore, the prosthetic rehabilitation can restore aesthetics, self-esteem, chewing and speech, but depending on their structural characteristics, not always do these functions return to the same standard present in the natural dentition, requiring an adjustment period.

\section{Consent}

Written informed consent was obtained from the patient for publication of this case report and any accompanying images.

\section{References}

[1] Migliorança, R.M., Coppedê, A., Rezende, R.C.L.D. and Mayo, T. (2011) Restoration of the Edentulous Maxilla Using Extrasinus Zygomatic Implants Combined with Anterior Conventional Implants: A Retrospective Study. The International Journal of Oral \& Maxillofacial Implants, 26, 665-672.

[2] Roumanas, E.D. (2009) The Social Solution-Denture Esthetics, Phonetics, and Function. Journal of Prosthodontics, 18, 112-115. https://doi.org/10.1111/j.1532-849X.2009.00440.x

[3] Aparício, C., Manresa, C., Francisco, K., Claros, P., Alández, J., Martin, O.G. and Albrektsson, T. (2014) Zygomatic Implants: Indications, Techniques and Outcomes, 
and the Zygomatic Success Code. Periodontology, 66, 41-58.

https://doi.org/10.1111/prd.12038

[4] Wang, F., Monje, A., Lin, G.H., Wu, Y., Monje, F., Wang, H.L. and Davó, R. (2015) Reliability of Four Zygomatic Implant-Supported Prostheses for the Rehabilitation of the Atrophic Maxilla: A Systematic Review. The International Journal of Oral \& Maxillofacial Implants, 30, 293-298. https://doi.org/10.11607/jomi.3691

[5] Sartori, E.M., Padovan, L.E.M., Sartori, I.A.M., Ribeiro Jr., P.L., Carvalho, A.C.G.S. and Goiato, M.C. (2012) Evaluation of Satisfaction of Patients Rehabilitated with Zygomatic Fixtures. Journal of Oral and Maxillofacial Surgery, 70, 314-319. https://doi.org/10.1016/j.joms.2011.03.044

[6] Kato, Y., Kizu, Y., Tonogi, M., Ide, Y. and Yamane, G.Y. (2005) Internal Structure of Zygomatic Bone Related to Zygomatic Fixture. Journal of Oral and Maxillofacial Surgery, 63, 1325-1329. https://doi.org/10.1016/j.joms.2005.05.313

[7] Sharma, A. and Rahul, G.R. (2013) Zygomatic Implants/Fixture: A Systematic Review. Journal of Oral Implantology, 39, 215-224.

https://doi.org/10.1563/AAID-JOI-D-11-00055

[8] Bertolai, R., Aversa, A., Catelani, C., Rossi, A. and Giannini, D. (2015) Treatment of Extreme Maxillary Atrophy with Zygoma Implants. Minerva Stomatologica, 64, 253-264.

[9] Branemark, P.I., Grondahl, K., Ohrnell, L.O., Nilsson, P., Petruson, B., Svensson, B., Engstrand, P. and Nannmar, U. (2004) Zygoma Fixture in the Management of Advanced Atrophy of the Maxilla: Technique and Long-Term Results. Scandinavian Journal of Plastic and Reconstructive Surgery and Hand Surgery, 38, 70-85. https://doi.org/10.1080/02844310310023918

[10] Bedrossian, E. and Stumpel, L.J. (2002) The Zygomatic Implant: Preliminary Data on Treatment of Severely Resorbed Maxillae. A Clinical Report. The International Journal of Oral \& Maxillofacial Implants, 17, 861-865.

[11] Bothur, S. and Garsten, M. (2010) Initial Speech Problems in Patients Treated with Multiple Zygomatic Implants. The International Journal of Oral \& Maxillofacial Implants, 25, 379-384. 